\title{
Blocking and self-locking of superdislocations in intermetallics
}

\author{
B. A. Greenberg ${ }^{1} \&$ M. A. Ivanov ${ }^{2}$ \\ ${ }^{1}$ Institute of Metal Physics, Ural Division, Russian Academy of Sciences, \\ Ekaterinburg, Russia \\ ${ }^{2}$ Institute of Metal Physics, National Academy of Sciences, Kiev, Ukraine
}

\begin{abstract}
Superdislocations are carriers of plastic deformation in intermetallics. A large translation vector, different types of stacking faults and antiphase boundaries determine the diversity of dislocation configurations, both glissile and blocked ones. A significant point is that blocked superdislocations, which are formed due to re-splitting of glissile superdislocations or rearrangement of the superpartial dislocation core, have the lowest energy. A new concept about the possibility of thermally activated blocking of superdislocations in the absence of external stresses (self-locking) was proposed. A sufficiently general thermally activated process, which causes the extension of a dislocation in a preferred direction and constitutes a necessary step in dislocation transformations leading to blocking, was revealed. By its nature, this process represents the flip of a dislocation from a shallow valley to a deep valley of the potential relief. Reasons for the multivalley relief and the presence of preferred directions vary for dislocations of different types in different materials. Consecutive stages of the rearrangement of an initial dislocation include the formation of a double kink and its subsequent reorientation along a preferred direction. The driving force of the process was calculated and conditions for its realization in the cases of perfect, superpartial and partial dislocations were formulated. An experimental proof of the proposed concept was obtained: self-locking of dislocations, which were induced by preliminary deformation, was detected in $\mathrm{Ni}_{3}(\mathrm{Al}, \mathrm{Nb})$ and $\mathrm{TiAl}$ during no-load heating.
\end{abstract}

Keywords: dislocations, plastic deformation, potential relief, shallow valley, deep valley, dislocation blocking, self-locking, no-load heating. 


\section{Introduction}

Although dislocation blocking mechanisms, which determine the deformation behavior of materials, are diverse, they can be divided into two groups. In the first group point blocking is due to pinning centers. In the second group linear blocking is explained by different factors, such as dislocation reactions, collisions of dislocations with domain boundaries and, finally, dislocation inherent transformations [1]. Such transformations, which are inherent in dislocations as linear defects, just represent the subject of this study. They are accomplished without participation of other dislocations and result from the rearrangement of the core of a perfect dislocation (BCC metals, TiAl - a single dislocation) or a partial dislocation (semiconductors). In some high-temperature intermetallics such transformations also result from re-splitting of a perfect or a superpartial dislocation [2]. Regardless of transformation details, a common feature is that the dislocation energy is gained at the expense of the dislocation mobility: a glissile dislocation turns to a dislocation barrier, which either remains indestructible or, under certain conditions, can re-transform to a glissile configuration. The barrier axis is the preferential direction along which the transformation to a low-energy configuration is realized. As a result, the potential relief is a multi-valley one for a dislocation: deep valleys extend in the preferential direction and shallow valleys go in other directions (fig. 1). Valleys of different depth along different directions can be distinguished (unlike fig. 1) in a three-dimensional display of the potential relief.

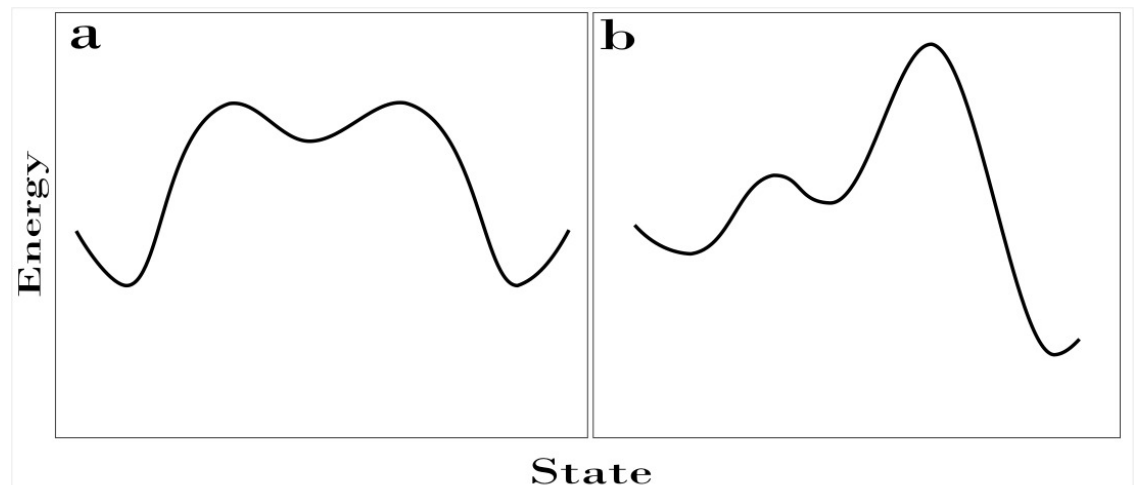

Figure 1: Schematic image of the potential relief; shallow valleys and deep valleys of one type (a) or two types (b).

\section{Flip-process}

The flip of a dislocation from a shallow to a deep valley of the potential relief, which causes the extension of the dislocation in the preferential direction, is a sufficiently general thermally activated process and constitutes a necessary step in dislocation transformations leading to blocking. 


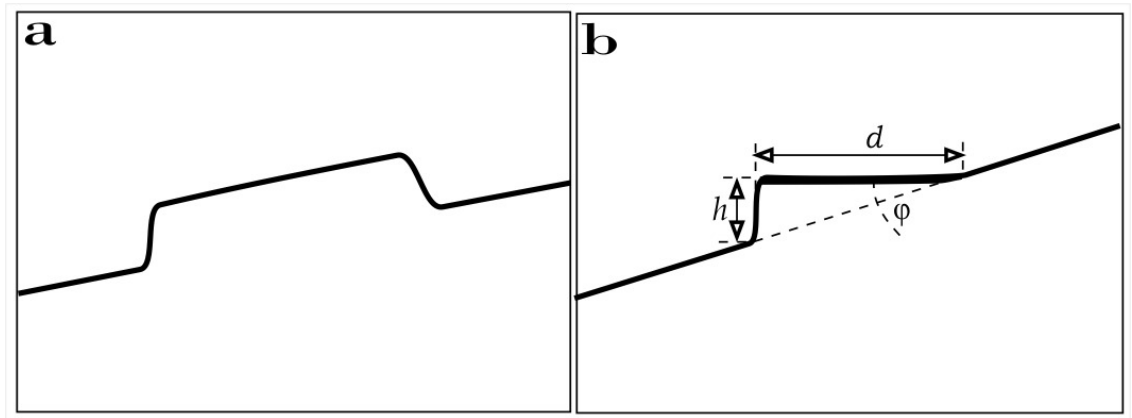

Figure 2: Consecutive stages of the rearrangement of the initial dislocation whose direction is close to the preferential direction: a - double kink; $b$ - reorientation in the preferential direction.

The flip process includes the formation of a double kink (fig. 2a) and its subsequent reorientation in the preferential direction resulting in the formation of an asymmetric kink (fig. 2b). The internal structure of the dislocation changes in the preferential direction and, hence, the dislocation energy decreases. Because this process takes place at different points along the dislocation line, the initial dislocation is broken down into long blocked segments. The flip process and the subsequent transfer from a deep to another deep valley ultimately determine the temperature dependence of the yield stress, $\sigma y(T)$. If the release from deep valleys is possible, $\sigma \mathrm{y}(\mathrm{T})$ will exhibit a normal behavior. If such release is hampered (indestructible barriers), an anomalous trend of $\sigma \mathrm{y}(\mathrm{T})$ will be observed in certain conditions.

According to Indenbom et al. [3], a double kink may be viewed as the nucleus of a "new phase" corresponding to the transition to a neighboring valley. We think that a chain [4] of asymmetric kinks rather than a single kink can be formed near the preferential direction (fig. 3a). This chain may be considered as a nucleus capable of transforming to a segment extended along the preferential direction and a multiple kink (fig. 3b). In any case, the extension of a dislocation in the preferential direction is a thermally activated process since it includes formation and propagation of kinks.

\section{Nucleation and propagation of kinks}

\subsection{Perfect dislocations}

Let us consider a potential relief of the following form: shallow valleys and a deep valley in some preferential direction, with the deep valley separated from the nearest shallow valley by a potential barrier. We shall assume for simplicity that the initial direction and the preferential direction are almost parallel. The double kink consists of initial dislocation segments located in a shallow valley, a segment of the length $d$ flipped to the deep valley, and single kinks connecting 
these segments. According to [5], the energy of the double kink can be written as the sum of the following contributions (with the corresponding signs): the double energy of a single kink; the work of the external stress $\sigma$ consumed for the formation of the double kink; and the interaction energy of the kinks. Furthermore, it is necessary to consider the change of the linear energy as the dislocation is transferred from a shallow valley to the deep valley. This contribution, which is connected with different depth of the valleys, was disregarded earlier and distinguishes the present study from other investigations. The critical configuration of the double kink, which is determined from conditions of the total energy extremum, has the length $d_{\mathrm{c}}$ equal to

$$
\begin{gathered}
d_{c}=\sqrt{\frac{K a^{2}}{(\sigma b a+\Delta E)},} \\
\Delta E=E_{0}-E_{v},
\end{gathered}
$$

Here $E_{0}$ is the energy of the dislocation in a shallow valley, $E_{\mathrm{v}}$ is the energy of the dislocation in the deep valley (both energies per unit length of the dislocation), $K=k \mu b^{2}, \mu$ is the shear modulus, and $k$ is a coefficient depending on the dislocation orientation.

If the external stress is not applied, the unstable configuration, which causes dispersion of the kinks, appears, as can be seen from (1), due to an additional driving force proportional to $\Delta E$. If $\sigma=0$, the condition for the flip process, which causes autoblocking of the dislocation, is the inequality $\Delta E>0$.

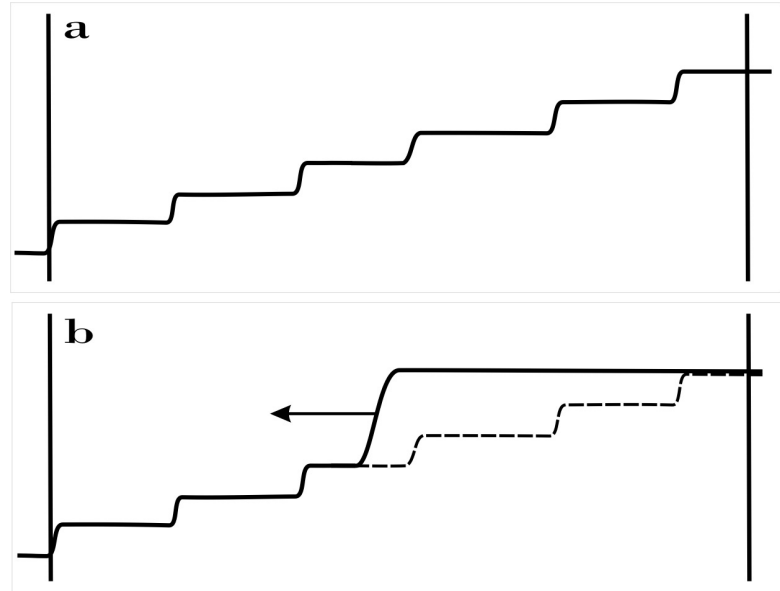

Figure 3: Chain of asymmetric kinks (a) and its transformation (b).

Obviously, this driving force simultaneously counteracts the reverse transition from a deep valley to a shallow valley. Therefore, thermally activated formation of indestructible barriers can be expected during no-load heating. 
Self-locking is still possible if directions, along which shallow and deep valleys are extended, are not parallel, but the angles between them are not too large. However, the critical configuration is not formed at large angles, because the energy loss during the kink spreading is not compensated by the energy gain during the dislocation flip to a deep trap. Let us estimate, rather roughly, the possibility that the configuration, which appears after the double kink reorientation (fig. 2b), develops or, oppositely, collapses. We shall assume for simplicity that a single kink is perpendicular to the preferential direction. Then the condition of the self-locking is that the energy of the dislocation, which is a broken line and consists of a segment of the length $d$ extended in the preferential direction and a single kink of the length $h$, is smaller than the energy of the initial rectilinear dislocation of the length $l$. This condition can be written as

$$
E_{\mathrm{v}} d+E_{0} h<E_{0} l \text {. }
$$

Introduce the angle $\varphi$ between the preferential direction and the direction of the initial dislocation (fig. 2b). Using (2) and writing $E_{\mathrm{v}}$ as $E_{\mathrm{v}}=E_{0}-\Delta E$ where $\Delta E>0$ is assumed, we obtain from (3):

$$
E_{0}(1+\operatorname{tg} \varphi-1 / \cos \varphi)<\Delta E .
$$

Thus, the condition of self-locking has the form

$$
\Delta E / E_{0}>\frac{\cos \varphi+\sin \varphi-1}{\cos \varphi} .
$$

It can be easily shown that the condition (5) is fulfilled at angles $\varphi<\tilde{\varphi}$, where $\tilde{\varphi}$ is determined from the equation

$$
\Delta E / E_{0}=\frac{\cos \tilde{\varphi}+\sin \tilde{\varphi}-1}{\cos \tilde{\varphi}} .
$$

Actually, $\tilde{\varphi}$ is the limiting angle for auto-locking. If $\varphi>\tilde{\varphi}$, this process is impossible, naturally in terms of the given model and the adopted approximations.

\subsection{Partial and superpartial dislocations}

It is possible that not perfect, but partial dislocations sink into a deep valley and just partial dislocations have the preferential direction. In this case, the development of the partial dislocation in the deep valley requires an additional energy proportional to the area of the stacking fault. Then, instead of (3), the condition of self-locking takes the form

$$
\left(E_{\mathrm{v}}+E_{0} \operatorname{tg} \varphi+1 / 2 \gamma d \operatorname{tg} \varphi\right) d<\left(E_{0} / \cos \varphi\right) d,
$$

where $\gamma$ is the stacking fault energy (per unit area).

The condition (5) changes correspondingly: 


$$
\Delta E / E_{0}>\frac{1}{\cos \varphi}\left(\cos \varphi+\sin \varphi\left(1+\frac{\gamma d}{2 E_{0}}\right)-1\right) .
$$

If we introduce the angle $\tilde{\varphi}$ by the relationship (6), then at $\varphi>\tilde{\varphi}$ and any $d$ the inequality

$$
\Delta E / E_{0}<\frac{1}{\cos \varphi}\left(\cos \varphi+\sin \varphi\left(1+\frac{\gamma d}{2 E_{0}}\right)-1\right), \varphi>\tilde{\varphi}
$$

is fulfilled.

This means that the condition (8) does not hold in this case and the flip of the partial dislocation to the deep valley is impossible at $\varphi>\tilde{\varphi}$ similarly to the perfect dislocation considered above.

If $\varphi<\tilde{\varphi}$, the condition (8) holds at small values of the segment length $d$. However, unlike for perfect dislocations, this condition no longer holds for partial dislocations as the length $d$ increases. The segment length $d_{\gamma}$, at which the condition becomes invalid, is defined by the relationship

$$
\Delta E / E_{0}=\frac{1}{\cos \varphi}\left(\cos \varphi+\sin \varphi\left(1+\frac{1}{2} \frac{\gamma}{E_{0}} d_{\gamma}\right)-1\right), \quad \varphi<\tilde{\varphi} .
$$

The existence of the physically reasonable solution of the equation (10) for $d_{\gamma}$ at a preset value of the angle $\varphi$ depends on the relationship between $\Delta E$ and $\gamma a$. To demonstrate this, we shall introduce the critical kink length $h_{\gamma}=d_{\gamma} \operatorname{tg} \varphi$. Obviously, the condition $h_{\gamma} / a>1$ should be fulfilled for the kink to exist in reality.

Using $h_{\gamma}$, the equation (10) can be rearranged to the form

$$
\frac{h_{\gamma}}{a}=\frac{\Delta E / E_{0}-(\cos \varphi+\sin \varphi-1) / \cos \varphi}{\gamma a / 2 E_{0}}, \quad \varphi<\tilde{\varphi}
$$

Let us consider the case when

$$
\Delta E<\gamma a .
$$

Then from (11) we have

$$
h_{\gamma} / a<1, \quad \varphi<\tilde{\varphi} .
$$

Hence, if the relationship (12) is fulfilled, a physically reasonable solution of the equation (11) is unavailable, i.e. self-locking does not take place.

Self-locking is possible only if the condition

$$
\Delta E>\gamma a
$$

is fulfilled. Moreover, it can be easily shown that the additional condition

$$
\varphi<\min \left\{\Delta E / E_{0}, \tilde{\varphi}\right\}
$$


should be met. Therefore, if the inequality (14) is fulfilled, the extension of segments, whose orientation satisfies (15), becomes possible.

The above expressions also hold when a preferential direction exists for superpartial dislocations connected by the APB band. It is assumed as before that kinks are formed independently (inconsistently) in each of the superpartial dislocations making up the superdislocation. Therefore, the nucleation and the propagation of kinks are determined by the relationship between $\Delta E$ and the APB energy in the corresponding plane.

\section{Examples}

\subsection{Blocking of a superpartial dislocation located initially in the cube plane $\left(\mathrm{Ni}_{3} \mathbf{A l}\right)$}

The initial superpartial dislocation is not splitted and, therefore, recombination is not required. The superpartial dislocation is blocked due to octahedral splitting. Since octahedral splitting is athermal, we have the only process that requires thermal fluctuations, namely the extension of the superpartial dislocation in the preferential direction. The preferential direction is a direction of the $<101>$ type parallel to the line of intersection between the cube and the octahedral plane. The energy gain from octahedral splitting determines $\Delta E$ and in the relationship (2) $E_{0}$ is the constricted dislocation energy and $E_{v}$ is the splitted dislocation energy. It is easily shown that $\Delta E$ can roughly be written as

$$
\begin{aligned}
& \Delta E \cong \psi\left(\boldsymbol{\beta}_{1}, \boldsymbol{\beta}_{2}\right) \ln \frac{d_{\mathrm{csf}}}{r_{0}}-\gamma_{\mathrm{csf}} d_{\mathrm{csf}}=\psi\left(\boldsymbol{\beta}_{1}, \boldsymbol{\beta}_{2}\right)\left(\ln \frac{d_{\mathrm{csf}}}{r_{0}}-1\right) \\
& \psi\left(\boldsymbol{\beta}_{1}, \boldsymbol{\beta}_{2}\right)=\frac{\mu}{2 \pi(1-v)}\left(\mathbf{e}_{1} \mathbf{e}_{2}+(1-v) \mathbf{s}_{1} \mathbf{s}_{2}\right)
\end{aligned}
$$

Here $\gamma_{\text {csf }}$ is the energy of a complex stacking fault, $d_{\text {csf }}$ is the equilibrium splitting width of the superpartial dislocation, $\mathbf{e}$ and $\mathbf{s}$ denote respectively the edge and the screw component of the Burgers vector $\boldsymbol{\beta}$ of the partial dislocation, $r_{0}$ is the dislocation core radius, and $v$ is the Poisson ratio.

The limiting angle $\tilde{\varphi}$ for self-locking is determined, as before, from the relationship (6). In this case, the energy $\gamma$ should be replaced by the APB energy $\zeta^{\prime}$ in the cube plane in the inequality (7) and the subsequent expressions containing $\gamma$. If the relationship

$$
\Delta E>\varsigma^{\prime} a,
$$

which is analogous to (14), is fulfilled, the self-locking process becomes possible. 
Indeed, experiments on no-load heating of $\mathrm{Ni}_{3}(\mathrm{Al}, \mathrm{Nb})$ single crystals predeformed at a high temperature demonstrated that superdislocations, which initially glided in the cube plane, turned to dislocation barriers $[6,7]$.

\subsection{Blocking of a superpartial dislocation located initially in the octahedral plane $\left(\mathrm{Ni}_{3} \mathrm{Al}\right)$}

A superpartial dislocation is blocked due to a series of consecutive transformations, including the cross slip of the superpartial dislocation from the octahedron to the cube plane, and its octahedral splitting. At each stage the superpartial dislocation extends in the preferential direction of the $<101>$ type, which is parallel to the line of intersection between the octahedron and the cube plane of the cross slip.

The effective force $K_{\left(\mathbf{b}, \mathbf{n}^{\prime}\right)}^{\text {eff }}$ providing the cross slip of the superpartial dislocation has the form [8]:

$$
K_{\left(\mathbf{b}, \mathbf{n}^{\prime}\right)}^{e f f}=b \sigma f+\left(\alpha \varsigma-\varsigma^{\prime}\right),
$$

where $\alpha=1 / \sqrt{3}$ and $f$ is the coefficient dependent on the Schmid factors. It readily follows from (18) that the cross slip of a superpartial dislocation is possible at $\sigma=0$ too. Therefore, self-locking of a superpartial dislocation, which is initially located in the octahedral plane, is possible if two conditions

$$
\begin{gathered}
\alpha \zeta-\zeta^{\prime}>0, \\
\Delta E>\zeta^{\prime} a
\end{gathered}
$$

are fulfilled simultaneously.

The condition (19) ensures the transfer of a superpartial dislocation to the cube plane. If (19) is fulfilled, the component $\alpha \zeta$ of the elastic repulsion force in the cube plane due to another superpartial dislocation is larger than the surface tension $\zeta^{\prime}$. The condition (20) is responsible for the subsequent extension of the superpartial dislocation along the line of intersection between the cube and the octahedral plane.

Indeed, experiments on no-load heating of $\mathrm{Ni}_{3}(\mathrm{Al}, \mathrm{Nb})$ single crystals predeformed at a low temperature showed that superdislocations, which initially glided in the octahedron plane, transformed to dislocation barriers $[6,7]$.

\subsection{Blocking of a single dislocation (TiAl)}

A single dislocation in TiAl is not connected with either the APB band or the stacking fault band, or another dislocation capable of initiating its blocking. The transfer of a single dislocation from a shallow to a deep valley corresponds to the dislocation core rearrangement [9]. Considering the covalent-like character of Ti$\mathrm{Ti}$ bonds, one may think that a screw dislocation with a narrow core has the lowest energy, because such bonds can be restored thanks to a constricted core. The preferential direction is the $<110]$ direction parallel to the Burgers vector of the single dislocation. Deep valleys are located along the said $<110$ ] direction. 
In actuality, the situation is more complicated because several types, rather than one type, of deep valleys are available. The potential relief (fig. 1b) was reconstructed $[2,10]$ using data on the nonmonotonic temperature dependence of the yield stress $\sigma_{\mathrm{y}}(T)$ in TiAl. Of course, the reconstruction of the potential relief is rough, but it reflects the variety of the core structure. Different forms of the core of a single dislocation in TiAl were obtained from computer simulation, specifically ab-initio simulation [11]. Several types of blocked core structures were revealed in each case.

Experiments on no-load heating of pre-deformed TiAl were performed. Only curvilinear dislocations were observed after deformation at room temperature. Blocked single dislocations were observed after subsequent no-load heating.

\subsection{Extension of a dislocation in the preferential direction in elementary semiconductors}

Preferential directions are those of the $<101>$ type with deep valleys of the Peierls potential relief for $30-\mathrm{deg}$ and $90-\mathrm{deg}$ partial dislocations. The difference $\Delta E$ is determined by the decrease in the energy of the partial dislocation core, resulting from its reconstruction. The possibility of a partial dislocation extending in crystallographic directions depends on which of the relations (12) or (14) between $\Delta E$ and $\gamma a$ is fulfilled. All known experiments (see, for example, $[12,13])$, which revealed segmentation of dislocations in preferential directions, were performed under a load. The extension in crystallographic directions was not detected after no-load heating of Si crystals [14]. One may think therefore that the relationship (12) holds in elementary semiconductors.

\section{Summary and conclusion}

Some thermally activated process, which is a necessary step of dislocation transformations, was revealed. This process represents the flip of a dislocation from a shallow valley of the potential relief to a deep valley located along the preferential direction. The flip process involves the formation of a double kink and its subsequent reorientation. Conditions for realization of the flip process at a zero external stress, which ensure self-locking of dislocations, were determined. Theoretically predicted self-locking of dislocations was confirmed in experiments on no-load heating of intermetallics after their preliminary deformation. An explanation was proposed why blocking of partial dislocations in elementary semiconductors is impossible without an external stress.

\section{Acknowledgements}

This study was performed under the State contract No. 02.467.11.2007 and supported by a grant from RFBR (project No. 04-03-96008) and the program of the Presidium RAS (project No. 10). 


\section{References}

[1] Escaig, B., Dislocation splitting and the plastic glide process in crystals. J. de Physique Suppl., 35(7), pp. 151-166, 1974.

[2] Greenberg, B. A. \& Ivanov, M. A., Microstructure and Deformation Behavior of $\mathrm{Ni}_{3} \mathrm{Al}$ and TiAl Intermetallic Compounds, Ural Div. Rus. Akad. Sci: Ekaterinburg, pp. 88-102, 2002 (in Russian).

[3] Indenbom, V. L., Petukhov, B. V. \& Lothe, J., Dislocation motion over the Peierls barrier (Chapter 8). Elastic Strain Fields and Dislocation Mobility,. eds. V.L. Indenbom \& J. Lothe, Elsevier Science Publishers B.V. pp. 491-516, 1992.

[4] Seeger, A. \& Schiller P., Bildung und diffusion von kinken als grundprozess der versetzungsbewegung bei der messung der inneren reibung. Acta Met., 10(4), pp. 348-357, 1962.

[5] Duesbery, M. S., The influence of core structure on dislocation mobility. Phil. Mag., 19(159). pp. 501-526, 1969.

[6] Greenberg, B. A., Antonova, O. V., Ivanov, M. A., Patselov, A. M. \& Plotnikov, A. V., Some features of formation and destruction of dislocation barriers in intermetallics. II. Observation of blocking superdislocations after no-load heating. The Physics of Metals and Metallography, 102 (1) pp. 69-76, 2006.

[7] Greenberg, B. A., Ivanov, M. A., Antonova, O. V., Patselov, A. M. \& Plotnikov, A. V., Deformation behavior of intermetallics: models and experiments. Proc. of the $4^{\text {th }}$ Int. Conf. On Mathematical Modelling and Computer Simulation of Material Technologies. Ariel, Israel, 1, pp. 122131, 2006.

[8] Greenberg, B. A. \& Ivanov, M. A., Some features of formation and destruction of dislocation barriers in intermetallics. I. Theory. The Physics of Metals and Metallography, 102 (1), pp. 61-68, 2006.

[9] Greenberg, B. A., Anisimov, V. I., Gornostirev Yu. N., Taluts G. G. Possible Factors Affecting the Brittleness of the Intermetallic Compound TiAl. II. Peierls Manyvalley Relief. Scripta Metall., 22 (6), pp. 859-864, 1988.

[10] Greenberg, B. A., Antonova, O. V., Volkov, A. Yu. \& Ivanov, M. A., The non-monotonic temperature dependence of the yield stress in TiAl and $\mathrm{CuAu}$ alloys. Intermetallics, 8, pp. 845-853, 2000.

[11] Woodward, C. \& Rao, S. I., Ab-initio of $(\mathrm{a} / 2<110]$ screw dislocations in $\gamma$-TiAl. Phil. Mag., 84 (3-5), pp. 401-413, 2004.

[12] Alexander, H., Dislocations in covalent crystals (Chapter 35). Dislocations in Solids, ed. F.R.N. Nabarro, Elsevier Sci. Publ. B.V. pp. 113-234, 1986.

[13] Rabier, J., George, A. Dislocations and plasticity in semiconductors. Revue Phys. Appl., 22(11), pp. 1327-1351, 1987.

[14] Patel, J. R. \& Kimerling, L. C., Dislocation defect states in Si. $J$ de Physique. Suppl., 40(6), pp. 67-70, 1979. 